\title{
FREQUENCY ANALYSIS OF CRACKED FUNCTIONALLY GRADED CANTILEVER BEAM
}

\author{
Nguyen Ngoc Huyen ${ }^{1}$, Nguyen Tien Khiem ${ }^{2, ~ * ~}$ \\ ${ }^{1}$ ThuyLoi University, 175 Tay Son, Dong Da, Hanoi \\ ${ }^{2}$ Vietnam Academy of Science and Technology, 18 Hoang Quoc Viet, Cau Giay, Hanoi \\ *Email:ntkhiem@imech.ac.vn; khiemvch@gmail.com
}

Received: 3 May 2016; Accepted for publication: 1 November 2016

\begin{abstract}
In this paper, a functionally graded cantilever beam with an open crack is investigated on the base of Timoshenko beam theory; power law of functionally graded material (FGM) and taking into account actual position of neutral axis instead of the central one. The open and edge crack is modeled by coupled translational and rotational springs stiffness of which is calculated by the formulas conducted accordingly to fracture mechanics. Using the frequency equation obtained in the framework of the theory natural frequencies of the beam are examined along the crack parameters and material properties. This analysis demonstrates that sensitivity of natural frequencies of FGM beam to crack is strongly dependent on the material constants of FGM.
\end{abstract}

Keywords: FGM, Timoshenko beam; cracked beam, modal analysis;

\section{INTRODUCTION}

Due to advantage properties compared to the laminate composites the functionally graded material (FGM) has been intensively studied recently and got wide application in the high-tech industries. An overview of the problems for manufacturing, modelling and testing FGM was given in [1]. Numerous methods such as the Finite Element Method (FEM) [2]; Spectral Element Method (SEM) [3]; Dynamic Stiffness Method (DSM) [4] or Rayleigh-Ritz method [5] have been developed for analysis of structures made of FGM. Nevertheless, the analytical methods are still the most accurate and efficient for dynamic analysis of functionally graded beam-like structures [6-9]. While the most of the aforementioned studies investigated undamaged beam, the crack problem in FGM has been studied in [10-11]. The most important result of the studies is that a crack in FGM beam can be modeled by an equivalent spring of stiffness calculated from the crack depth. Based on the rotational spring model of crack, Yang and Chen [12] studied free vibration and buckling of Euler-Bernoulli FGM beam with edge cracks. They found that natural frequencies of FGM beam with smaller slenderness and lower ratio of the bottom Young's modulus to the top one are more sensitive to cracks. The transfer matrix method was employed by Wei et al. [13] for obtaining frequency equation of FGM beam 
with arbitrary number of cracks in the form of third-order determinant. This simplifies significantly the modal analysis of multiple cracked FGM beam. Aydin [14] has conducted an expression for mode shape of FGM beam with multiple cracks and used it for constructing the frequency equation in the form of an explicit determinant of third-order also. Forced vibration and nonlinear free vibration of cracked FGM beam are investigated in Ref. [15-16]. Based on the exponential law of FGM and rotational spring model of crack, Yu and Chu [17] and Banerjee et al. [18] have applied the FEM and the Frequency Contour Method (FCM) for detecting a crack in Euler-Bernoulli and Timoshenko FGM beams, respectively. Nguyen Tien Khiem and Nguyen Ngoc Huyen obtained a condition for uncoupling of longitudinal and bending vibration in FGM beam and studied uncoupled flexural vibration of the beam [19].

In the present paper, an analytical approach in frequency domain is proposed to study free vibration of functionally graded Timoshenko beam with an open crack modeled by a pair of translational and rotational springs. This is a novelty of present paper in comparison with the previous ones where only rotational spring model of crack was adopted. Using the proposed model of crack, frequency equation of a cracked cantilever is conducted and used for sensitivity analysis of natural frequencies to crack parameters. Numerical results of natural frequencies as functions of crack positions and depths are obtained by MATLAB code.

\section{GOVERNING EQUATIONS}

\subsection{Model of FGM beam}

Consider a beam of length $L$, cross-section area $A=b \times h$ made of FGM with material parameters varying along thickness by the power law

$$
\left\{\begin{array}{l}
E(z) \\
G(z) \\
\rho(z)
\end{array}\right\}=\left\{\begin{array}{l}
E_{b} \\
G_{b} \\
\rho_{b}
\end{array}\right\}+\left\{\begin{array}{l}
E_{t}-E_{b} \\
G_{t}-G_{b} \\
\rho_{t}-\rho_{b}
\end{array}\right\}\left(\frac{z}{h}+\frac{1}{2}\right)^{n},-h / 2 \leq z \leq h / 2,
$$

where $E, G$ and $\rho$ with indexes $t$ and $b$ stand for elasticity, shear modulus and material density at the top and bottom respectively; $z$ is ordinate from the central axis at high $h / 2$. Assuming linear theory of shear deformation, the displacement fields in the cross-section at $x$ are

$$
u(x, z, t)=u_{0}(x, t)-\left(z-h_{0}\right) \theta(x, t) ; w(x, z, t)=w_{0}(x, t),
$$

with $u_{0}(x, t), w_{0}(x, t)$ being the displacements of neutral axis that is located at the high $h_{0}$ from the central axis; $\theta$ is slope of the cross-section. Therefore, constituting equations get the form

$$
\varepsilon_{x}=\partial u_{0} / \partial x-\left(z-h_{0}\right) \partial \theta / \partial x ; \gamma_{x z}=\partial w_{0} / \partial x-\theta
$$

and

$$
\sigma_{x}=E(z) \varepsilon_{x} ; \tau_{x z}=\kappa G(z) \gamma_{x z} .
$$

In the latter equation $\kappa$ is a coefficient introduced to account for the geometry-dependent distribution of shear stress. Hamilton principle allows one to obtain equations of motion in the time domain as

$$
I_{11} \ddot{u}-A_{11} u^{\prime \prime}-I_{12} \ddot{\theta}=0
$$




$$
\begin{gathered}
I_{12} \ddot{u}-I_{22} \ddot{\theta}+A_{22} \theta^{\prime \prime}+A_{33}\left(w^{\prime}-\theta\right)=0 ; \\
I_{11} \ddot{w}-A_{33}\left(w^{\prime \prime}-\theta^{\prime}\right)=0,
\end{gathered}
$$

where

$$
\begin{gathered}
A_{11}=\frac{2 E_{0} A\left(R_{e}+n\right)}{\left(R_{e}+1\right)(1+n)} ; I_{11}=\frac{2 \rho_{0} A\left(R_{\rho}+n\right)}{\left(R_{\rho}+1\right)(1+n)} ; I_{12}=\frac{2 \rho_{0} A h}{\left(R_{\rho}+1\right)}\left[\frac{2 R_{\rho}+n}{2(2+n)}-\frac{R_{\rho}+n}{(1+n)} \alpha\right] ; \\
A_{22}=\frac{24 E_{0} I_{0}}{\left(R_{e}+1\right)}\left[\frac{3 R_{e}+n}{3(3+n)}-\frac{2 R_{e}+n}{(2+n)} \alpha+\frac{R_{e}+n}{(1+n)} \alpha^{2}\right] ; \\
A_{33}=\frac{2 \kappa G_{0} A\left(R_{G}+n\right)}{\left(R_{G}+1\right)(1+n)} ; \\
I_{22}=\frac{24 \rho_{0} I_{0}}{\left(R_{\rho}+1\right)}\left[\frac{3 R_{\rho}+n}{3(3+n)}-\frac{2 R_{\rho}+n}{(2+n)} \alpha+\frac{R_{\rho}+n}{(1+n)} \alpha^{2}\right] ; \alpha=1 / 2+h_{0} / h ; \quad(2.6) \\
h_{0}=\frac{n\left(R_{e}-1\right) h}{2(n+2)\left(n+R_{e}\right)}, R_{e}=\frac{E_{t}}{E_{b}}, R_{\rho}=\frac{\rho_{t}}{\rho_{b}} ; E_{0}=\frac{E_{b}+E_{t}}{2} ; \rho_{0}=\frac{\rho_{b}+\rho_{t}}{2} ; G_{0}=\frac{G_{b}+G_{t}}{2} ; \\
I_{0}=b h^{3} / 12 ; A=b h .
\end{gathered}
$$

Introducing the displacement amplitudes

$$
\{U, \Theta, W\}=\int_{-\infty}^{\infty}\left\{u_{0}(x, t), \theta(x, t), w_{0}(x, t)\right\} e^{-i \omega t} d t
$$

Eq. (2.5) get to be

$$
\begin{gathered}
\left(\omega^{2} I_{11} U+A_{11} U^{\prime \prime}\right)-\omega^{2} I_{12} \Theta=0 ; \\
\left(\omega^{2} I_{22} \Theta+A_{22} \Theta^{\prime \prime}\right)-\omega^{2} I_{12} U+A_{33}\left(W^{\prime}-\Theta\right)=0 ; \\
\omega^{2} I_{11} W+A_{33}\left(W^{\prime \prime}-\Theta^{\prime}\right)=0 .
\end{gathered}
$$

Using the following matrix and vector notations

$$
\begin{gathered}
\mathbf{A}=\left[\begin{array}{ccc}
A_{11} & 0 & 0 \\
0 & A_{22} & 0 \\
0 & 0 & A_{33}
\end{array}\right] ; \mathbf{\Pi}=\left[\begin{array}{ccc}
0 & 0 & 0 \\
0 & 0 & A_{33} \\
0 & -A_{33} & 0
\end{array}\right] ; \\
\mathbf{C}(\omega)=\left[\begin{array}{ccc}
\omega^{2} I_{11} & -\omega^{2} I_{12} & 0 \\
-\omega^{2} I_{12} & \omega^{2} I_{22}-A_{33} & 0 \\
0 & 0 & \omega^{2} I_{11}
\end{array}\right] ; \\
\mathbf{z}=\{U, \Theta, W\}^{T},
\end{gathered}
$$

Eq. (2.8) are rewritten in the form [19] 


$$
A z^{\prime \prime}+\Pi z^{\prime}+\mathbf{C z}=0
$$

\subsection{Crack modeling}

Assume that the beam has been cracked at the position $e$ measured from the left end of beam and the crack is modeled by a pair of equivalent springs of stiffness $T$ for translational spring and $R$ for rotational one. Therefore, conditions that must be satisfied at the crack are

$$
\begin{gathered}
U(e+0)-U(e-0)=N(e) / T ; \Theta(e+0)-\Theta(e-0)=M(e) / R ; W(e+0)=W(e-0) ; \\
N(e)=N(e+0)=N(e-0) ; Q(e+0)=Q(e-0) ; M(e+0)=M(e-0)=M(e),(2.10)
\end{gathered}
$$

where $N, Q, M$ are respectively internal axial, shear forces and bending moment at section $x$

$$
N=A_{11} U_{x}^{\prime} ; M=A_{22} \Theta_{x}^{\prime} ; Q=A_{33}\left(W_{x}^{\prime}-\Theta\right) .
$$

Substituting (2.11) into (2.10) one can rewrite the latter conditions as

$$
\begin{gathered}
U(e+0)=U(e-0)+\gamma_{1} U_{x}^{\prime}(e) ; \Theta(e+0)=\Theta(e-0)+\gamma_{2} \Theta_{x}^{\prime}(e) ; W(e+0)=W(e-0) ; \\
U_{x}^{\prime}(e+0)=U_{x}^{\prime}(e-0) ; \Theta_{x}^{\prime}(e+0)=\Theta_{x}^{\prime}(e-0) ; W_{x}^{\prime}(e+0)=W_{x}^{\prime}(e-0)+\gamma_{2} \Theta_{x}^{\prime}(e), \\
\gamma_{1}=A_{11} / T ; \gamma_{2}=A_{22} / R .
\end{gathered}
$$

The so-called crack magnitudes $\gamma_{1}, \gamma_{2}$ introduced in (2.13) are function of the material parameters such as elastic modulus and they should be those of homogeneous beam when $E_{t}=E_{b}=E_{0}$. On the other hand, using expressions (2.6) the crack magnitudes (2.13) can be rewritten as

$$
\gamma_{1}=\gamma_{a} \theta_{1}\left(R_{E}, n\right) ; \gamma_{2}=\gamma_{b} \theta_{2}\left(R_{E}, n\right)
$$

where

$$
\begin{aligned}
\gamma_{a} & =E_{0} A / T ; \gamma_{b}=E_{0} I_{0} / R \\
\theta_{1}=\frac{2\left(R_{e}+n\right)}{\left(R_{e}+1\right)(1+n)} ; \theta_{2} & =\frac{24}{\left(R_{e}+1\right)}\left[\frac{3 R_{e}+n}{3(3+n)}-\frac{2 R_{e}+n}{(2+n)} \alpha+\frac{R_{e}+n}{(1+n)} \alpha^{2}\right] .
\end{aligned}
$$

In case of homogeneous beam when $R_{e}=1$ the crack magnitudes must be equal to $\gamma_{10}, \gamma_{20}$, that are calculated from crack depth $a$ for axial [20] and flexural [21] vibrations as

$$
\begin{gathered}
\gamma_{10}=E_{0} A / T_{0}=2 \pi\left(1-v_{0}^{2}\right) h f_{1}(z), z=a / h \\
f_{1}(z)=z^{2}\left(0.6272-0.17248 z+5.92134 z^{2}-10.7054 z^{3}+31.5685 z^{4}-67.47 z^{5}+\right. \\
\left.+139.123 z^{6}-146.682 z^{7}+92.3552 z^{8}\right) \\
\gamma_{20}=E_{0} I / R_{0}=6 \pi\left(1-v_{0}^{2}\right) h f_{2}(z) \\
f_{2}(z)=z^{2}\left(0.6272-1.04533 z+4.5948 z^{2}-9.9736 z^{3}+20.2948 z^{4}-33.0351 z^{5}+\right. \\
\left.+47.1063 z^{6}-40.7556 z^{7}+19.6 z^{8}\right)
\end{gathered}
$$


For modal analysis of cracked FGM beam crack magnitudes are proposed herein to be approximately calculated using expressions (2.16-2.17) with $\gamma_{a}=\gamma_{10}, \gamma_{b}=\gamma_{20}$, i. e.

$$
\begin{gathered}
\gamma_{1}=F_{1}(a) ; \gamma_{2}=F_{2}(a) ; \\
F_{1}(a)=2 \pi\left(1-v_{0}^{2}\right) h \theta_{1} f_{1}(a) ; F_{2}(a)=6 \pi\left(1-v_{0}^{2}\right) h \sigma_{2} f_{2}(a) .
\end{gathered}
$$

These functions would be used for calculating the crack magnitudes from given crack depth.

\subsection{Characteristic equation}

Continuous solution of Eq. (2.9) sought in the form $\mathbf{z}_{0}=\mathbf{d} e^{\lambda x}$ yields the equation

$$
\left[\lambda^{2} \mathbf{A}+\lambda \mathbf{\Pi}+\mathbf{C}\right] \mathbf{d}=0 .
$$

The latter equation would have nontrivial solution with respect to constant vector $\mathbf{d}$ under the condition

$$
\operatorname{det}\left[\lambda^{2} \mathbf{A}+\lambda \mathbf{\Pi}+\mathbf{C}\right]=0
$$

that can be in turn expressed in the form

$$
\left.\left(\lambda^{2} A_{11}+\omega^{2} I_{11}\right)\left[\left(\lambda^{2} A_{33}+\omega^{2} I_{11}\right)\left(\lambda^{2} A_{22}+\omega^{2} I_{22}\right)-\omega^{2} I_{11} A_{33}\right)\right]-\left(\lambda^{2} A_{33}+\omega^{2} I_{11}\right) \omega^{4} I_{12}^{2}=0 .
$$

This is in fact a cubic equation with respect to $\eta=\lambda^{2}$ that could be elementarily solved and results in three roots $\eta_{1}, \eta_{2}, \eta_{3}$. Introducing the notations

$$
\lambda_{1,4}= \pm k_{1}= \pm \sqrt{\eta_{1}} ; \lambda_{2,5}= \pm k_{2}= \pm \sqrt{\eta_{2}} ; \lambda_{3,6}= \pm k_{3}= \pm \sqrt{\eta_{3}}
$$

general continuous solution of Eq. (2.9) is represented as

$$
\mathbf{z}_{0}(x, \omega)=\mathbf{G}(x, \omega) \mathbf{C},
$$

with $\mathbf{C}=\left(C_{1}, \ldots, C_{6}\right)^{T}=\left(d_{11}, \ldots, d_{16}\right)^{T}$ and

$$
\begin{gathered}
\mathbf{G}(x, \omega)=\left[\mathbf{G}_{1}(x, \omega) \mathbf{G}_{2}(x, \omega)\right] ; \\
\mathbf{G}_{1}(x, \omega)=\left[\begin{array}{ccc}
\alpha_{1} e^{k_{1} x} & \alpha_{2} e^{k_{2} x} & \alpha_{3} e^{k_{3} x} \\
e^{k_{1} x} & e^{k_{2} x} & e^{k_{3} x} \\
\beta_{1} e^{k_{1} x} & \beta_{2} e^{k_{2} x} & \beta_{3} e^{k_{3} x}
\end{array}\right] ; \mathbf{G}_{2}(x, \omega)=\left[\begin{array}{ccc}
\alpha_{1} e^{-k_{1} x} & \alpha_{2} e^{-k_{2} x} & \alpha_{3} e^{-k_{3} x} \\
e^{-k_{1} x} & e^{-k_{2} x} & e^{-k_{3} x} \\
-\beta_{1} e^{-k_{1} x} & -\beta_{2} e^{-k_{2} x} & -\beta_{3} e^{-k_{3} x}
\end{array}\right], \\
\alpha_{j}=\frac{\omega^{2} I_{12}}{\omega^{2} I_{11}+\lambda_{j}^{2} A_{11}} ; \beta_{j}=\frac{\lambda_{j} A_{33}}{\left(\omega^{2} I_{11}+\lambda_{j}^{2} A_{33}\right)} ; j=1,2,3 .
\end{gathered}
$$

Using (2.22), it can be found that solution of Eq. (2.9) denoted by $\mathbf{S}(x)$ satisfying the conditions

$$
\mathbf{S}(0)=\left(S_{1}^{0}, S_{2}^{0}, 0\right)^{T} ; \mathbf{S}^{\prime}(0)=\left(0,0, S_{3}^{0}\right)^{T}
$$

is represented as

$$
\mathbf{S}(x)=[\boldsymbol{\Phi}(x)]\left\{\mathbf{S}^{0}\right\}
$$


where $\mathbf{S}^{0}=\left\{S_{1}^{0}, S_{2}^{0}, S_{3}^{0}\right\}^{T}$ and matrix

$$
\begin{gathered}
{[\boldsymbol{\Phi}(x)]=\left[\begin{array}{ccc}
\alpha_{1} \cosh k_{1} x & \alpha_{2} \cosh k_{2} x & \alpha_{3} \cosh k_{3} x \\
\cosh k_{1} x & \cosh k_{2} x & \cosh k_{3} x \\
\beta_{1} \sinh k_{1} x & \beta_{2} \sinh k_{2} x & \beta_{3} \sinh k_{3} x
\end{array}\right] \cdot\left[\begin{array}{ccc}
\delta_{11} & \delta_{12} & \delta_{13} \\
\delta_{21} & \delta_{22} & \delta_{23} \\
\delta_{31} & \delta_{32} & \delta_{33}
\end{array}\right] ;} \\
\Delta_{1}=\left(\delta_{11} S_{1}^{0}+\delta_{12} S_{2}^{0}+\delta_{13} S_{3}^{0}\right) / \Delta ; \Delta_{2}=\left(\delta_{21} S_{1}^{0}+\delta_{22} S_{2}^{0}+\delta_{23} S_{3}^{0}\right) / \Delta ; \\
\Delta_{3}=\left(\delta_{31} S_{1}^{0}+\delta_{32} S_{2}^{0}+\delta_{33} S_{3}^{0}\right) / \Delta ; \Delta=k_{1} \beta_{1}\left(\alpha_{2}-\alpha_{3}\right)+k_{2} \beta_{2}\left(\alpha_{3}-\alpha_{1}\right)+k_{3} \beta_{3}\left(\alpha_{1}-\alpha_{2}\right) ;
\end{gathered}
$$

Assuming furthermore that $S_{1}^{0}=\gamma_{1} U_{x}^{\prime}(e), S_{2}^{0}=S_{3}^{0}=\gamma_{2} \Theta_{x}^{\prime}(e)$ or $\mathbf{S}^{0}=[\boldsymbol{\Sigma}]\left\{\mathbf{z}_{0}^{\prime}(e)\right\}$ with

$$
\boldsymbol{\Sigma}=\left[\begin{array}{ccc}
\gamma_{1} & 0 & 0 \\
0 & \gamma_{2} & 0 \\
0 & \gamma_{2} & 0
\end{array}\right]
$$

a particular solution $\mathbf{z}_{c}(x)$ of Eq. (2.9) that satisfies initial conditions

$$
\mathbf{z}_{c}(0)=\left\{\gamma_{1} U_{x}^{\prime}(e), \gamma_{2} \Theta_{x}^{\prime}(e), 0\right\}^{T} ; \mathbf{z}_{c}^{\prime}(0)=\left(0,0, \gamma_{2} \Theta_{x}^{\prime}(e)\right)^{T}
$$

is

$$
\mathbf{z}_{c}(x)=[\boldsymbol{\Phi}(x)][\boldsymbol{\Sigma}]\left\{\mathbf{z}_{0}^{\prime}(e)\right\}=\left[\mathbf{G}_{c}(x)\right]\left\{\mathbf{z}_{0}^{\prime}(e)\right\} .
$$

Using the matrix-function notation

$$
\mathbf{K}(x)=\left\{\begin{array}{c}
\mathbf{G}_{c}(x): x>0 \\
0 \quad: x \leq 0 ;
\end{array} \mathbf{K}^{\prime}(x)=\left\{\begin{array}{r}
\mathbf{G}_{c}^{\prime}(x): x>0 \\
0: x \leq 0
\end{array}\right.\right.
$$

one is able to prove that the function

$$
\mathbf{z}(x)=\mathbf{z}_{0}(x)+\mathbf{K}(x-e) \mathbf{z}_{0}^{\prime}(e)
$$

is general solution of Eq. (2.9) satisfying conditions (2.12) at the cracked section.

It can be easily to verify that boundary conditions for cantilever beam are

$$
u(0, t)=w(0, t)=\theta(0, t)=0 ; N(L, t)=M(L, t)=Q(L, t)=0 .
$$

Applying conditions (2.32a) for solution (2.31) leads to

$$
\begin{gathered}
\mathbf{B}_{01} \mathbf{C}_{1}+\mathbf{B}_{02} \mathbf{C}_{2}=0, \\
\mathbf{C}_{1}=\left\{C_{1}, C_{2}, C_{3}\right\}^{T} ; \mathbf{C}_{2}=\left\{C_{4}, C_{5}, C_{6}\right\}^{T} ; \\
\mathbf{B}_{01}=\left[\begin{array}{ccc}
\alpha_{1} & \alpha_{2} & \alpha_{3} \\
1 & 1 & 1 \\
\beta_{1} & \beta_{2} & \beta_{3}
\end{array}\right] ; \mathbf{B}_{02}=\left[\begin{array}{ccc}
\alpha_{1} & \alpha_{2} & \alpha_{3} \\
1 & 1 & 1 \\
-\beta_{1} & -\beta_{2} & -\beta_{3}
\end{array}\right] .
\end{gathered}
$$

Obviously, the above equation allows the vectors $\mathbf{C}_{1}, \mathbf{C}_{2}$ to be expressed as

$$
\mathbf{C}_{1}=\left[\mathbf{B}_{01}\right]^{-1} \mathbf{D}, \mathbf{C}_{2}=-\left[\mathbf{B}_{02}\right]^{-1} \mathbf{D}
$$


with an arbitrary constant vector $\mathbf{D}$, so that solution $\mathbf{z}_{0}(x)$ can be rewritten in the form

$$
\mathbf{z}_{0}(x, \omega)=\mathbf{G}_{0}(x, \omega) \mathbf{D},
$$

where

$$
\mathbf{G}_{0}(x, \omega)=\mathbf{G}_{1}(x, \omega) \mathbf{B}_{01}^{-1}-\mathbf{G}_{2}(x, \omega) \mathbf{B}_{02}^{-1} .
$$

Consequently, one obtains

$$
\mathbf{z}(x)=\left[\mathbf{G}_{0}(x, \omega)+\mathbf{K}(x-e) \mathbf{G}_{0}^{\prime}(e, \omega)\right]\{\mathbf{D}\}=\left[\mathbf{G}_{\mathrm{L}}(x, \omega)\right]\{\mathbf{D}\} .
$$

Applying boundary condition (3.32b) for solution (2.34) one gets

$$
\begin{gathered}
\left.\left[\mathbf{B}_{\mathrm{LL}} \omega\right)\right]\{\mathbf{D}\}=0 \\
\mathbf{B}_{\mathrm{LL}}(\omega)=\left.\mathbf{B}_{\mathrm{L}}\left\{\mathbf{G}_{\mathrm{L}}(x, \omega)\right\}\right|_{x=L} ; \mathbf{B}_{L}=\left[\begin{array}{ccc}
A_{11} \partial_{x} & 0 & 0 \\
0 & A_{22} \partial_{x} & 0 \\
0 & -A_{33} & A_{33} \partial_{x}
\end{array}\right] .
\end{gathered}
$$

So that characteristic or frequency equation of the cracked FGM beam is obtained as

$$
\Lambda(\omega)=\operatorname{det}\left[\mathbf{B}_{\mathrm{LL}}(\omega)\right]=0 .
$$

Positive root $\omega_{j}$ of this equation provide desired natural frequency of the beam. In the case of intact beam the frequency equation (2.37) is reduced to

$$
\begin{aligned}
\Lambda_{0}(\omega) & =\operatorname{det}\left[\mathbf{B}_{\mathrm{L} 0}(\omega)\right]=0 . \\
\mathbf{B}_{\mathrm{L} 0}(\omega) & =\left.\mathbf{B}_{\mathrm{L}}\left\{\mathbf{G}_{0}(x, \omega)\right\}\right|_{x=L} .
\end{aligned}
$$

Thus, forward problem is to calculate natural frequencies of cracked or uncracked FGM beam by solving Eq. (2.37) or (2.38).

\section{NUMERICAL RESULTS AND DISCUSSION}

\subsection{Comparative study}

To investigate effect of actual position of neutral axis on natural frequencies of Timoshenko FGM cantilevered beam, it is examined an undamaged beam studied in [4] that is composed from steel: $E_{b}=210 G P a, \rho_{b}=7800 \mathrm{~kg} / \mathrm{m}^{3}, \mu_{1}=0.3$ at the bottom and Aluminum Oxide $\left(\mathrm{Al}_{2} \mathrm{O}_{3}\right): E_{t}=390 G P a, \rho_{t}=3960 \mathrm{~kg} / \mathrm{m}^{3}, \mu_{t}=0.25$ at the top surface.

Tables 1 shows first five natural frequencies computed in the present paper for various slenderness ratio $L / h$, and power law index $n$. Comparison with those obtained in [4] where neutral axis is assumed coincident with the middle one shows that effect of actual position of neutral axis on the lower natural frequencies is clearly observed in the case of small slenderness, $\mathrm{L} / \mathrm{h}=5$, and $\mathrm{n}=2$. In this case natural frequencies calculated with actual position of neutral axis are lower than those computed by the centroid axis theory. However, higher natural frequencies of FGM beam with greater slenderness and power law index are not very much changed by the correcting position of neutral axis. 
Table 1. Comparison of frequency parameters, $\lambda=\left(\omega L^{2} / h\right) \sqrt{\rho_{b} / E_{b}}$, for undamaged FGM cantilever beam: Present - actual and Ref. [4] - centroid position of neutral axis.

\begin{tabular}{|c|c|c|c|c|c|c|c|c|c|}
\hline \multicolumn{2}{|c|}{$L / h$} & \multicolumn{2}{|c|}{5} & \multicolumn{2}{|c|}{10} & \multicolumn{2}{|c|}{20} & \multicolumn{2}{|c|}{30} \\
\hline$n$ & $\begin{array}{l}\text { Fr. } \\
\text { No. }\end{array}$ & Present & Ref.[4] & Present & Ref.[4] & Present & Ref.[4] & Present & Ref.[4] \\
\hline \multirow{5}{*}{0.1} & 1 & 1.7377 & 1.7574 & 1.7854 & 1.7966 & 1.8020 & 1.8070 & 1.8060 & 1.8089 \\
\hline & 2 & 9.3254 & 9.0511 & 10.6630 & 10.782 & 11.1116 & 11.196 & 11.2359 & 11.278 \\
\hline & 3 & 14.1039 & 14.095 & 28.0582 & 28.190 & 30.4454 & 30.800 & 31.1476 & 31.325 \\
\hline & 4 & $\begin{array}{l}22.3755 \\
375464\end{array}$ & 22.682 & 28.3600 & 28.404 & 56.3576 & 56.379 & 60.2240 & 60.681 \\
\hline & 5 & & 37.747 & 51.8239 & 51.618 & 58.0334 & 58.897 & 84.5711 & 84.569 \\
\hline \multirow{5}{*}{0.2} & 1 & 1.6294 & 1.6638 & 1.6804 & 1.7010 & 1.7011 & 1.7107 & 1.7061 & 1.7126 \\
\hline & 2 & 8.6806 & 8.9969 & 9.9804 & 10.208 & 10.4534 & 10.600 & 10.5981 & 10.678 \\
\hline & 3 & 13.4167 & 13.390 & 26.3172 & 26.781 & 28.5635 & 29.161 & 29.3406 & 29.657 \\
\hline & 4 & 20.9419 & 21.482 & 26.9510 & 26.895 & 53.4530 & 53.562 & 56.6568 & 57.449 \\
\hline & 5 & 35.5698 & 35.754 & 48.9926 & 48.878 & 54.4140 & 55.762 & 80.3494 & 80.343 \\
\hline \multirow{5}{*}{0.5} & 1 & 1.4308 & 1.4911 & 1.4852 & 1.5244 & 1.5118 & 1.5332 & 1.5183 & 1.5348 \\
\hline & 2 & 7.5158 & 8.0609 & 8.7058 & 9.1477 & 9.2390 & 9.4992 & 9.4075 & 9.5691 \\
\hline & 3 & 12.0814 & 12.012 & 22.8654 & 24.024 & 25.1327 & 26.130 & 25.9870 & 26.576 \\
\hline & 4 & $\begin{array}{l}18.3974 \\
32.0833\end{array}$ & 19.243 & 24.5762 & 24.098 & 47.3355 & 48.048 & 50.0731 & 51.475 \\
\hline & 5 & 32.0833 & 32.022 & 43.5626 & 43.787 & 48.3416 & 49.962 & 72.0863 & 72.072 \\
\hline \multirow{5}{*}{1.0} & 1 & 1.2809 & 1.3557 & 1.3345 & 1.3864 & 1.3636 & 1.3945 & 1.3705 & 1.3960 \\
\hline & 2 & 6.6597 & 7.3164 & 7.7397 & 8.3146 & 8.3071 & 8.6383 & 8.4791 & 8.7027 \\
\hline & 3 & 10.9037 & 10.811 & 20.3079 & 21.623 & 22.5403 & 23.755 & 23.3925 & 24.165 \\
\hline & 4 & 16.4188 & 17.441 & 22.1864 & 21.886 & 42.3703 & 43.246 & 45.0165 & 46.795 \\
\hline & 5 & 28.9477 & 28.989 & 38.7308 & 39.732 & 43.5214 & 45.402 & 64.8906 & 64.870 \\
\hline \multirow{5}{*}{2.0} & 1 & 1.1757 & 1.2471 & 1.2252 & 1.2762 & 1.2519 & 1.2839 & 1.2583 & 1.2853 \\
\hline & 2 & 6.1047 & 6.7053 & 7.1063 & 7.6440 & 7.6240 & 7.9501 & 7.7835 & 8.0112 \\
\hline & 3 & 9.8238 & 9.7403 & 18.6028 & 19.481 & 20.6777 & 21.851 & 21.4688 & 22.239 \\
\hline & 4 & 15.0301 & 15.937 & 20.0358 & 20.088 & 38.6088 & 38.961 & 41.3021 & 43.049 \\
\hline & 5 & 26.4566 & 26.428 & 35.5854 & 36.403 & 39.4505 & 41.733 & 58.4651 & 58.442 \\
\hline \multirow{5}{*}{5.0} & 1 & 1.1030 & 1.1446 & 1.1405 & 1.1722 & 1.1604 & 1.1795 & 1.1651 & 1.1809 \\
\hline & 2 & 5.8414 & 6.1274 & 6.6706 & 7.0111 & 7.0955 & 7.3014 & 7.2209 & 7.3594 \\
\hline & 3 & 8.8103 & 8.7633 & 17.3374 & 17.527 & 19.2998 & 20.057 & 19.9462 & 20.425 \\
\hline & 4 & 14.3168 & 14.516 & 17.7729 & 18.391 & 35.0124 & 35.053 & 38.4225 & 39.525 \\
\hline & 5 & 24.9321 & 24.009 & 32.4530 & 33.2625 & 36.5838 & 38.278 & 52.5932 & 52.580 \\
\hline \multirow{5}{*}{10} & 1 & 1.0629 & 1.0867 & 1.0962 & 1.1130 & 1.1105 & 1.1199 & 1.1138 & 1.1212 \\
\hline & 2 & 5.6345 & 5.8159 & 6.4753 & 6.6562 & 6.8192 & 6.9324 & 6.9167 & 6.9876 \\
\hline & 3 & 8.3591 & 8.3430 & 16.6488 & 16.686 & 18.6108 & 19.394 & 19.1378 & 19.394 \\
\hline & 4 & 13.5189 & 13.776 & 17.0828 & 17.459 & 33.3597 & 33.372 & 36.9246 & 37.532 \\
\hline & 5 & 22.8182 & 22.783 & 31.2177 & 31.575 & 35.3365 & 36.345 & 50.0635 & 50.058 \\
\hline
\end{tabular}



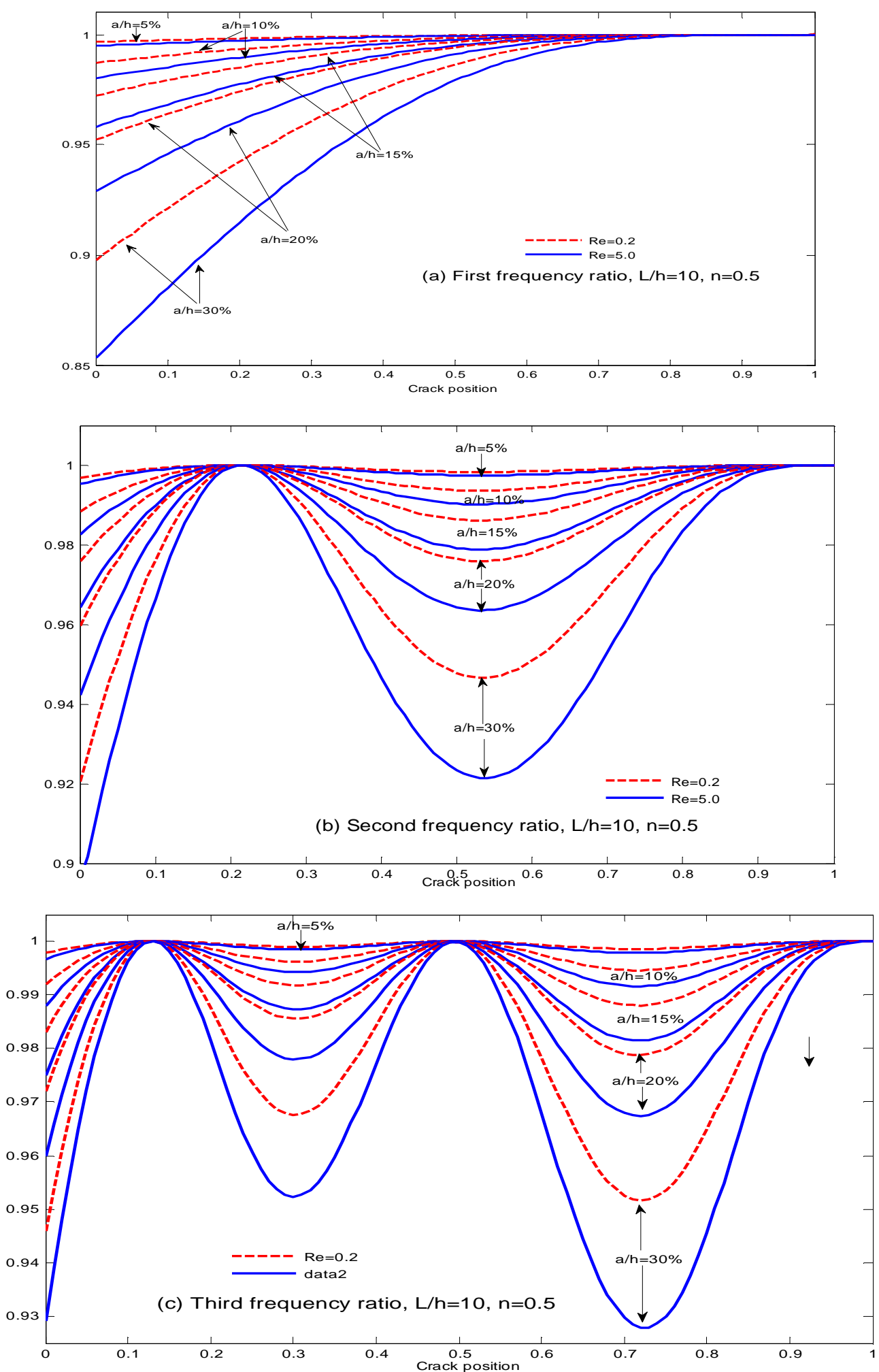

Figure 1. Sensitivity of natural frequencies (a- first, b- second, c- third) in dependence on crack depth $(5 \%-30 \%)$ and elasticity modulus ratio $\mathrm{R}_{\mathrm{e}}=0.2 \& 5.0$ with $\mathrm{L} / \mathrm{h}=10, \mathrm{n}=5$. 


\subsection{Sensitivity of natural frequencies to crack}
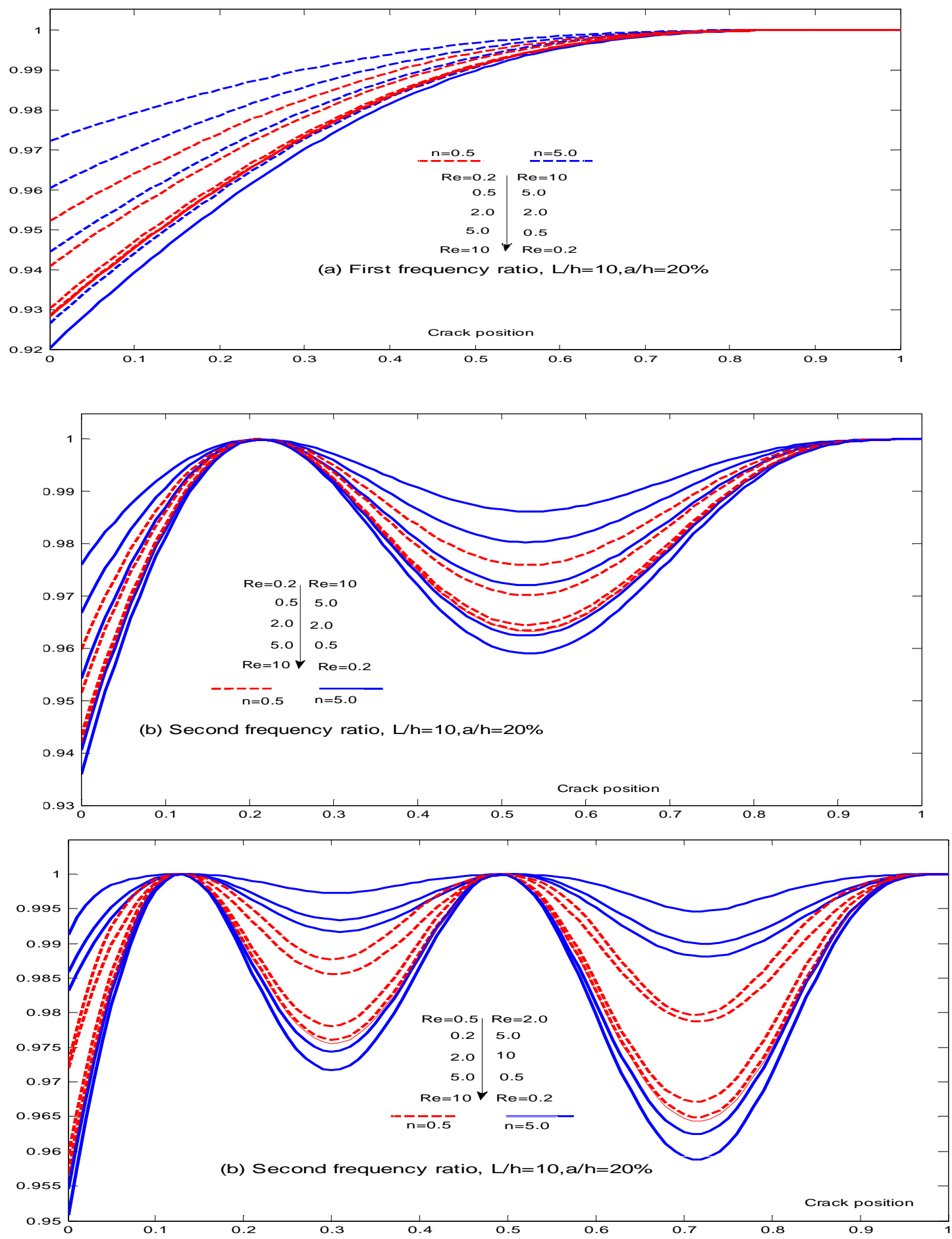

Figure 2. Sensitivity of natural frequencies (a- first, b- second, c - third) in dependence on the elasticity modulus ratio $R_{e}(0.1-10)$ and $\mathrm{n}=0.5 ; 5.0$ with $\mathrm{L} / \mathrm{h}=10$, a/h=20\%. 
The change in natural frequencies caused by a crack is usually called sensitivity of the natural frequencies to crack. The natural frequency sensitivity is represented in this paper by a ratio of the damaged to undamaged frequencies as function of crack location along the beam length. Such indicator for the natural frequency sensitivity is investigated herein in dependence on the material and geometry parameters of a FGM cantilever beam. Results are shown in Figs. 1-5 for combinations of various crack depth $\mathrm{a} / \mathrm{h}$, slenderness ratio $\mathrm{L} / \mathrm{h}$, power law index $n$ and elasticity modulus ratio $R_{e}$.

First, it is observed in the Figures that, likely to the homogeneous beam, a natural frequency could be unchanged if crack occurred at some positions on beam. Such positions are called critical points (or frequency node) for vibration mode with the unchanged frequency. For instance, the free end of homogeneous cantilever beam is a consistent critical point for all modes including either axial or flexural vibration. Approximate critical points for first three vibration modes with undamaged natural frequency $\omega_{k}^{0}$ of an FGM beam are given in Table 2.

Table 2. Possible critical points for FGM Timoshenko cantilever beam.

\begin{tabular}{|c|c|c|c|}
\hline Mode & First frequency node & Second frequency node & Third frequency node \\
\hline 1 & \multicolumn{3}{|c|}{} \\
\hline 2 & 0.22 & 1.0 & no \\
\hline 3 & 0.13 & 0.49 & 1.0 \\
\hline
\end{tabular}

Observation of the graphs given in Figs. 1-4 provide that the sensitivity of natural frequencies is monotonically reducing with growing crack depth and it is dependent also on the material and geometry of the beam. Namely, the sensitivity is increasing with elasticity modulus ratio $R_{E}=E_{t} / E_{b}$ for $n<1$ and decreasing when $n>1$. The latter implies that increase of elasticity modulus from bottom to top of Timoshenko beam makes the natural frequencies more or less sensitive to crack dependently on that $n<1$ or $n>1$. Similarly, it is observed from Fig. 3 that natural frequency sensitivity is increasing with $n$ for a fixed $R_{E}<1$ and would be decreasing if $R_{E}>1$. Fig. 4 shows that natural frequencies of flexural vibration modes become less sensitive to crack for increasing slenderness ratio and it is independent on whatever material the beam is made of but the axial mode frequencies show to be most sensitive to crack when $\mathrm{L} / \mathrm{h}$ $=10$. 

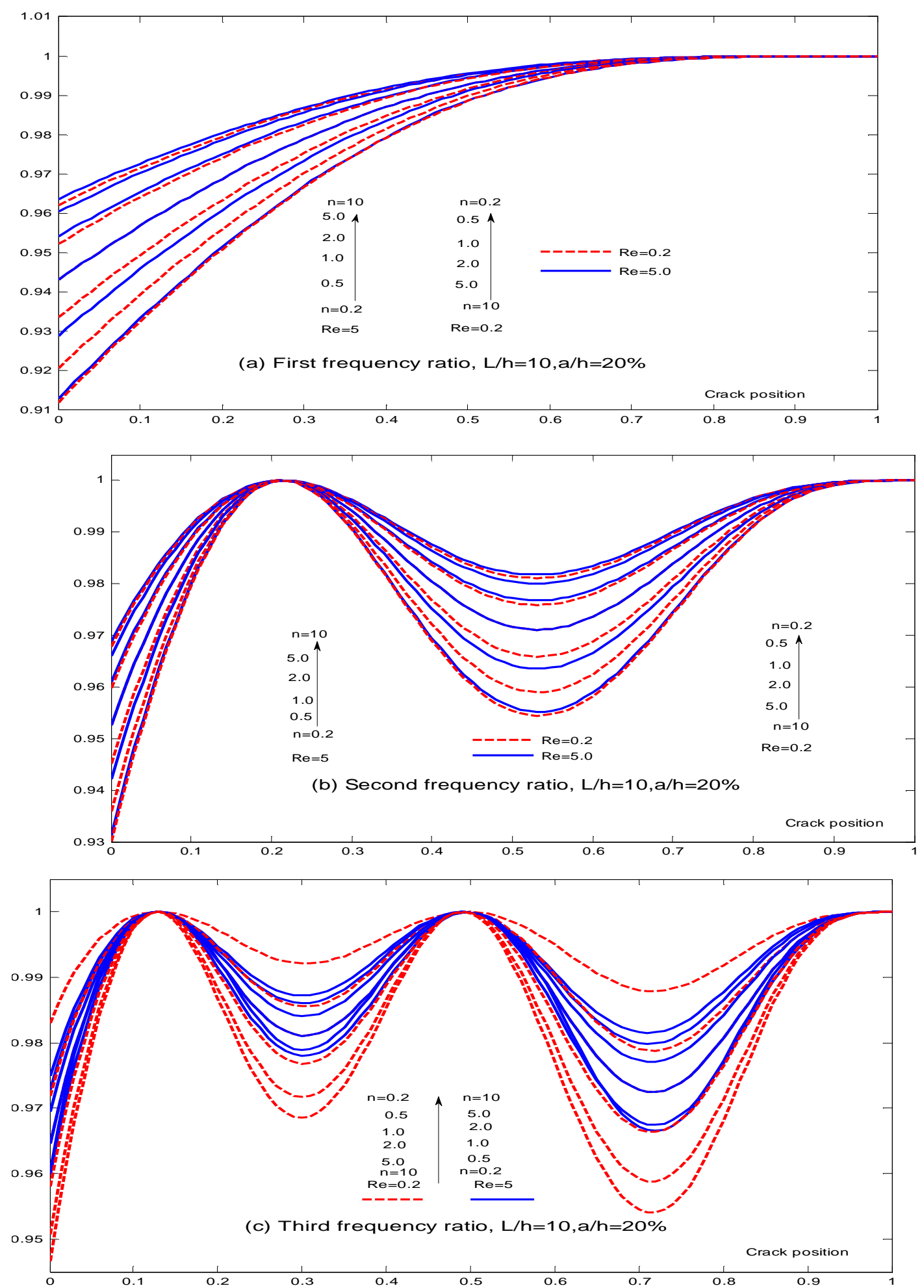

Figure 3. Sensitivity of natural frequencies (a- first, b- second, c-third) in dependence on the power law index $\mathrm{n}=0.2-10$; the slenderness ratio $\mathrm{L} / \mathrm{h} 10$ with elasticity modulus ratio $R_{e}=0.2 \& 5.0$ and crack depth $\mathrm{a} / \mathrm{h}=20 \%$. 

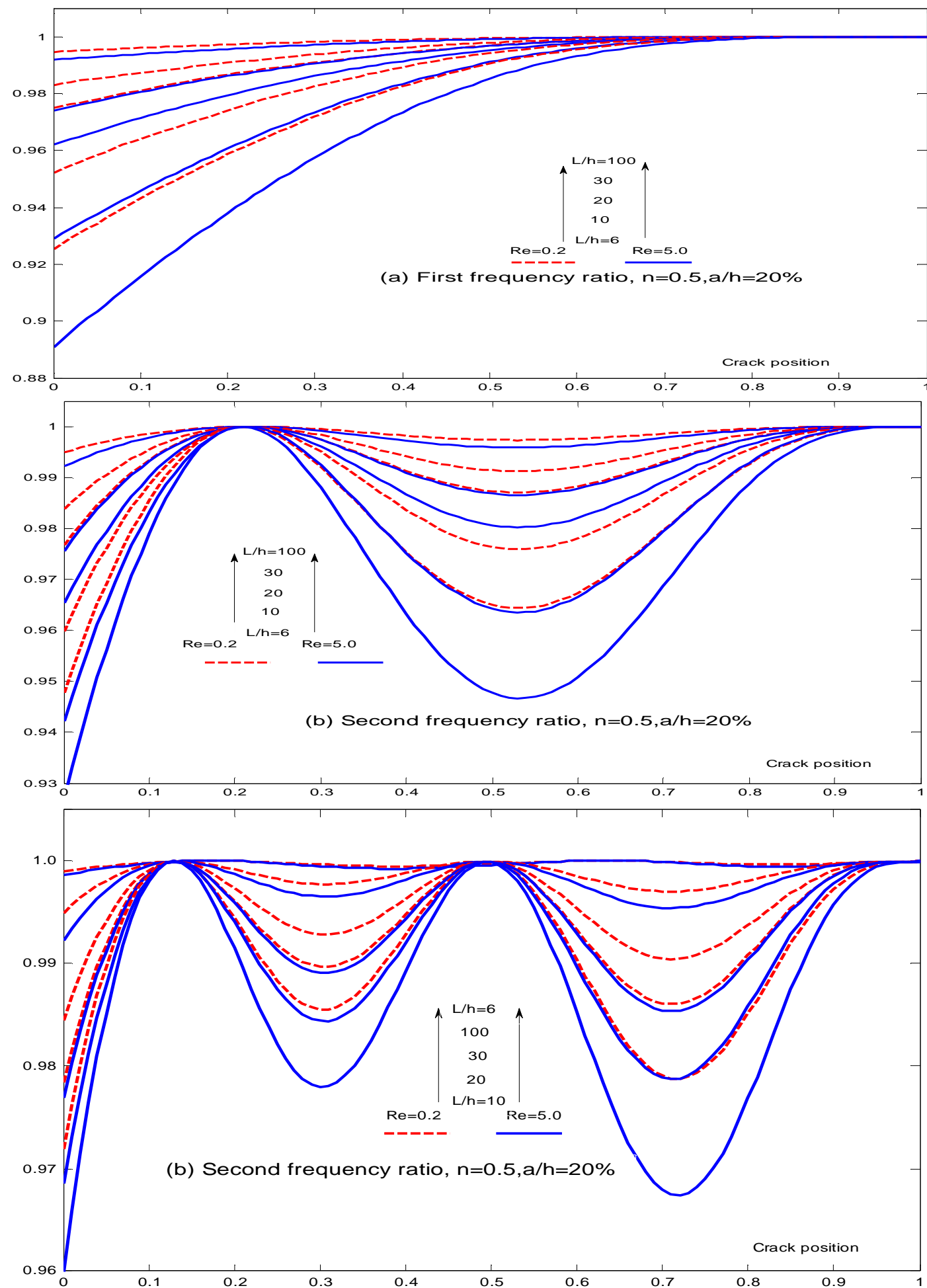

Figure 4. Sensitivity of natural frequencies (a- first, b- second, c-third) in dependence on the slenderness ratio $\mathrm{L} / \mathrm{h}=5-50$ with elasticity modulus ratio $R_{e}=0.2 \& 5.0, n=05 \& 5.0$ and crack depth a/h $=20 \%$. 


\section{CONCLUSION}

Major results obtained in the present paper are as follows:

A consistent theory of vibration beam has been formulated in the frequency domain for functionally graded Timoshenko beam that can be used for analysis of either free or forced vibrations in the beam.

Frequency equation for functionally graded Timoshenko beam with single crack modeled by coupled translation and rotation springs was constructed in a form that is applicable straightforward to frequency analysis of the beam. Application of the equation for natural frequency analysis of FGM beam demonstrates that natural frequencies of flexural vibration modes are more sensitive to crack than those of axial vibration modes and the natural frequency sensitivity is strongly dependent on both material and geometry parameters of functionally graded Timoshenko beam.

The theory proposed in the present work can be further developed for analysis and identification of FGM beam with multiple cracks.

Acknowledgement. The first author is sincerely thankful to the NAFOSTED of Vietnam for final support under Grant Number: 107.01-2015.20.

\section{REFERENCES}

1. Birman V. and Byrd L.W. - Modeling and Analysis of Functional Graded Materials and Structures. Applied Mechanics Reviews 60 (2007) 195-215.

2. Chakraborty A., Gopalakrishnan S. and Reddy J.N. - A new beam finite element for the analysis of functional graded materials. International Journal of Mechanical Science $\mathbf{4 5}$ (2003) 519-539.

3. Chakraborty A. and Gopalakrishnan S. - A spectrally formulated finite element for wave propagation analysis in functionally graded beams. International Journal of Solids and Structures 40 (2003) 2421-2448.

4. Su H. and Banerjee J.R. - Development of dynamic stiffness method for free vibration of functionally graded Timoshenko beams. Computers and Structures 147 (2015) 107-116.

5. Pradhan K.K. and Chakraverty S. - Free vibration of Euler and Timoshenko functionally graded beams by Rayleigh-Ritz method. Composite: Part B 51 (2013) 175-184.

6. Zhong Z. and Yu T. - Analytical solution of a cantilever functionally graded beam. Computer Science and Technology 67 (2007)481-488.

7. Sina S.A., Navazi H.M. and Haddadpour H. - An analytical method for free vibration analysis of functionally graded beams. Material and Design 30 (2009)741-747.

8. Li X.F. - A unified approach for analyzing static and dynamic behaviors of functionally graded Timoshenko and Euler-Bernoulli beams. Journal of Sound and Vibration 318 (2008)1210-1229.

9. Simsek M. and Kocatuk T. - Free and forced vibration of a functionally graded beam subjected a concentrated moving harmonic load. Composite Structures 90 (4) (2009) 465473. 
10. Jin Z.H. and Batra R.C. - Some basic fracture mechanics concepts in functionally graded materials. J. Mech. Phys. Solids 44 (8) (1996)1221-1235.

11. Erdogan F. and Wu B.H. - The surface crack problem for a plate with functionally graded properties. Journal of Applied Mechanics 64 (1997) 448-456.

12. Yang J. and Chen Y. - Free vibration and buckling analysis of functionally graded beams with edge cracks. Composite Structures 83 (2008) 48-60.

13. Wei D., Liu Y.H. and Xiang Z.H. - An analytical method for free vibration analysis of functionally graded beams with edge cracks. Journal of Sound and Vibration 331 (2012) 1685-1700.

14. Aydin K. - Free vibration of functional graded beams with arbitrary number of cracks. European Journal of Mechanics A/Solid 42 (2013) 112-124.

15. Kitipornchai S., Ke L.L., Yang J. and Xiang Y. - Nonlinear vibration of edge cracked functionally graded Timoshenko beams. Journal of Sound and Vibration 324 (2009) 962982.

16. Yan T., Kitipornchai S., Yang J. and He X. Q. - Dynamic behavior of edge-cracked shear deformable functionally graded beams on an elastic foundation under a moving load. Composite Structures 93 (2011) 2992-3001.

17. Yu Z. and Chu F. - Identification of crack in functionally graded material beams using the p-version of finite element method. Journal of Sound and Vibration 325 (2009) 69-85.

18. Banerjee A., Panigrahi B. and Pohit G. - Crack modelling and detection in Timoshenko FGM beam under transverse vibration using frequency contour and response surface model with GA. Nondestructive Testing and Evaluation 2015; DOI.10.1080/10589759.2015.1071812.

19. Khiem N. T., and Huyen N. N. - Uncoupled vibration in functionlly graded Timoshenko beam, Journal of Science and Technology 54 (6) (2016) 785.

20. Chondros T.G., Dimarogonas A.D. and Yao J. - Longitudinal vibration of a continous cracked bar. Engineering Fracture Mechanics 61 (1998) 593-606.

21. Chondros T.G. and Dimarogonas A.D. - A continuous cracked beam theory. Journal of Sound and Vibration 215 (1998) 17-34. 